\title{
PARADIGMS
}

\section{Consciousness and Quantum Information Processing: Uncovering the Foundation for a Medicine of Light}

\author{
BRUCE D. CURTIS, M.A., M.Div., ${ }^{1,2}$ \\ and J.J. HURTAK, Ph.D., (Social Science) Ph.D. (History \& Oriental Studies) ${ }^{1,3}$
}

\section{ABSTRACT}

Great progress has been made by Russian and German researchers in recent years (Popp, Voeikov, and others) to examine the biophysical aspects of biophotonic processes in humans. This paper suggests that there ํํㄴ a many-body reality to the way biophysical light interacts with the human self-organization of information tothat may be achieved by means of biomolecular, metabolic, or neural communication. These systems may merge Eas mobile energy relay systems similar to what is seen as qi processes in acupuncture science, suggesting a "holomovement" that seeks to confirm itself and increasingly retrieves and uses only the information that serves itts exchanges with the environment. This coevolution of evolutionary process levels, expressed in process terms, $\because$ can be seen as a foundation for a Medicine of Light integrating hidden variables in consciousness studies with functional differentiation and new findings in the biologic sciences.

"I regard consciousness as fundamental. I regard matter as a derivative of consciousness."

-Max Planck

\section{INTRODUCTION}

$\mathbf{T}$ he development of quantum theory in the early twentieth century and the elegant Einstein equivalence established once and for all that matter is energy. Perhaps this led Einstein to state enigmatically in 1916: "For the rest of my life I want to reflect on what light is." David Bohm (1980) also saw all of existence as an unending sea of light, a "holomovement" in which matter is a crystallized form of light. We too are left to reflect on what light itself is. Biologic science has been slow in the uptake, failing to appropriate the implications of quantum physics, and attempting to abstract bodies from their greater matrix of energy. Medical researchers and practitioners need not repeat this mistake, but can and should appropriate the knowledge of this dynamis for the healing sciences.

The encounter of the West with the ancient medical science of acupuncture and other studies in biophysics have awakened a deep curiosity about other possible modes of energy transmission and communication within the body, beyond the already known circulatory systems: the blood, the lymphatic, and the nervous system. Modern experimental work suggests that we are beginning to map some type of light circulatory system operating on an energetic level in a markedly different manner from that of its molecular counterparts. Recent studies by Zhang $(1996,2003)$ suggest an entire body distinct from the chemical body that interpenetrates it. Such a body has been termed the electromagnetic body and may be a vehicle of consciousness projection.

\footnotetext{
${ }^{1}$ The Academy for Future Science, Los Gatos, CA.

${ }^{2}$ Society for the Advancement of Global Education (S.A.G.E.), Oceanside, CA.

${ }^{3}$ Universidad Autonoma Metropolitana, Distrito Federal, Mexico.
} 
Researchers in the biologic sciences are beginning to come to terms with a complementary model of biologic functioning pioneered by Gurwitsch et al. (the Vernadsky-Gurwitsch-Bauer school). Gurwitsch $(1944,1948)$ introduced the concepts of the morphogenetic field (1944) and mitogenetic radiation (i.e., ultra-weak photon emission from living systems). Research continues with the work of Popp (1992), Voeikov (2000), van Wijk (2001), and others. It is a brave new world wherein the basis of life is located in the world of biophotons, electron excited states (EES), and quantum coherence; biologic systems functioning as lasers (Korotkov, 2002). At a deeper level, a truly holistic approach requires vacuum biophysics (Bischof, 2000) where the potential is as important as the actual (Dürr, 1998). These perspectives portray the body more as an energy biocomputer (Gariaev, 1994; Hurtak, 1973a) than a molecular machine. A system operating in on nonequilibrium thermodynamics (Beloussov and Popp, 1995; Prigogine, 1967), it is understood as open to fields ond energies that from the contemporary human perspecthive may seem new. These fields include consciousness fields of a mind reality operating beyond the functions of İneuronal interactions and microtubule structures (Stapp, डे 1993).

$\stackrel{\sigma}{\Xi}$ These new fields and new energy realities that have dawakened human scientific curiosity are more than passing energy phenomena; in a deeper sense they reflect the

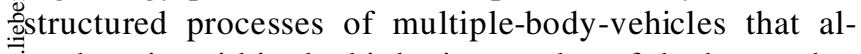
ready exist within the biologic complex of the human being. This paper looks at the interplay between the two: the consciousness and material realities. For if these other त̣models are true, holism will come to have an even more Țexpanded meaning, and holistic medicine will be comIpelled to include the many subtle consciousness and eninergy fields that interpenetrate and commingle to form the totality that we call the human being. In so doing, medi觉cine will evolve to draw on the full healing power implied J्डby the insight of physicist Bernard d'Espagnat, who said: ¿'The doctrine that the world is made up of objects whose existence is independent of human consciousness turns out to be in conflict with quantum mechanics and with facts established by experiment."

For the purpose of clear discussion in this paper, the authors propose the distinction between energy and consciousness, such that consciousness goes beyond the wave component of matter and is not reducible to information fields conceived as epiphenomena emerging from material structure that has reached a certain stage of complexity. Rather, consistent with Planck's insight quoted above, we view matter and energy as complementary aspects of information fields that emerge from consciousness, which is the source and ground of all that appears. As the universal and ubiquitous presence, consciousness is the hidden variable in the cosmos that in varying degrees, directs and participates in all quantum events. In sum, it is the core reality holding the entire multi-dimensional universe together in its unified field.*

The current effort on the part of the authors is aimed at devising a program that will illustrate, elucidate, and elaborate the basic assumptions of the human mind-matter transducer needed to encourage interest and research into consciousness functions that may operate above threshold levels in clinical contexts. In the process, there will of necessity be a languaging across disciplines, in order to come to grips with the emerging picture of many types of light, from the particulate (photonic) to the superluminal and consciousness light. Perhaps scientists will one day acknowledge, as mystics within all wisdom traditions have done, that within our consciousness can be found the cause of our disease and the source of our healing.

\section{BIOPHOTONICS AND THE STRUCTURING OF THE BODY'S ENERGY}

\section{Mitogenetic radiation and quantum coherence in the body}

Nobel laureate Dr. Albert Szent-Györgi (1960), father of modern biochemistry once stated that: "The cell is a machine driven by energy. It can thus be approached by studying matter or by studying energy. In every culture and in every medical tradition before ours, healing was accomplished by moving energy." This statement makes a methodological claim for moving our medical science into a more essential and holistic model of the human being and the healing process. We are compelled by its insight to examine the nature and types of energy, its forms, its origins, its transformations. In the first section we examine the generation of energy in the body by means distinct from the well-known process of adenosine triphosphate (ATP) production in the mitochondria via oxidative phosphorylation. We encounter another more powerful, and orderly or coherent source of energy for bodily function that is generated by the action of reactive oxygen species (ROS) in water, blood, and aqueous solutions (Voeikov, 2003a).

\footnotetext{
${ }^{*}$ More formal mathematical expression of these complex fields of intelligence was developed by the great mathematician Charles Musès, who built upon the work of William Rowan Hamilton, Eugene Wigner and others, and showed that quantum physics observationally requires the introduction of nonordinary, or nonreal numbers, beginning with so-called imaginary numbers based on i, $\sqrt{ }-1$. Even the simple representation of electromagnetic phenomena and their effects are impossible without the proper use of $\sqrt{ }-1$. The characterization of consciousness, particularly higher forms, along with altered states, is postulated as requiring even greater hypernumbers beyond this (Musès, 1972). It may be understood, along the lines of Erwin Shrödinger's profound realization, that individual consciousness is one with universal consciousness, as the drop of water is one with the ocean.
} 
The revolutionary research of Gurwitsch (1944) introduced the concepts of mitogenetic radiation (MGR) and the morphogenetic field. He proposed that: "[I]t is wrong to oppose the notion of a structure to the notion of a process. The only correct approach to living systems is an approach to them as to structured processes, flowing in molecular complexes widely different in the degree of their lability." MGR is a high-density energy transfer showing "action at a distance" on populations of cells and inducing a very specific response, namely cell division (Voeikov, 2003a). In short, MGR is the signaling, or triggering factor in mitosis, which Gurwitsch hypothesized cannot occur without the involvement of one photon of ultraviolet (UV) light. The biophotons necessary for mitosis are associated with cell growth and differentiation (Popp, 1979) and entirely distinct from bioluminescence. Furthermore, biophotons are mediators of zintercellular communication (Chang et al., 2000; Kaz-

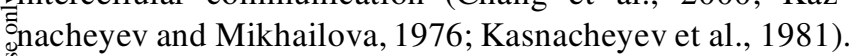
Understanding the biophotonic action of the body in both i. Smitosis and the bioinformational function (to be explored in greater depth in the next section) will enhance our grasp of Sthe human being as a complex and multidimensional conFfluence of diverse energy dynamics, possibly operating on Æparallel spectra.

Carefully examining the MGR process to lay the foundation for the structured organization of energy, Gurwitsch took .ّ ergy along common electronic levels of protein molecules could now be applied to what he called constellations or, in scurrent terms, ensembles; these are linked clusters of macroत̣molecules held together by constant energy circulation, seen त़as a common electronic cloud that is shared. Through this S. cloud energy states are transferred via local electrons, eliminating the need for electronic motion (Korotkov, 2002).

b Here we must understand the body as a thermodynamic

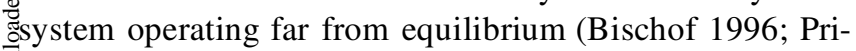
gogine 1967) exhibiting characteristics of chaos and structuring the energy for biophysical activity as a dissipative structure. Within this dynamic, the human system does not exclusively exhibit entropy, the second law of thermodynamics, but may also operate on the principle of centropy. Here we understand centropy to be the electrification of matter, wherein entropy is reversed and order increases. ${ }^{*}$ It should be noted that the photon emissions are ultra-weak, and thus we are led to the principle that it is not the intensity of the radiation, but the order that counts. Here we discover that coherence is the fundamental property of biophotons, where biologic efficiency increases with decreasing intensity (Voeikov, 2003). Remarkably, biophotons are extremely coherent, even more so than human-made lasers (Popp et al., 1992; van Wijk, 1993). Cyril Smith (2004) points out the revolutionary implications of quanta and coherence effects in water and living systems, experimentally demonstrating that information can be imprinted as fre- quencies by the interaction of quantum and electromagnetic (e-m) fields within water as well as more complex living systems. Observed microclusters in water are likely to be a result of such field imprints as the microscope photographs of Masaru Emoto portray strikingly (pp. 19-21).

Essential to the biophotonic process of energy transformations is the presence of ROS, particularly radical oxygen or $\mathrm{O}_{2}{ }^{-} \cdot$, the superoxide anion radical that initiates the production of all other ROS (Voeikov, 2000, 2001). A cascading process creates an abundance of organismal energy wherein recombination reactions lead to the storage of highly organized and structured energy in EES (Voeikov, 2003a). EES differ from energy of common cell resources generated by ATP. Actually, there is an order of magnitude difference in energy produced by these distinct processes, with energies released from ATP measuring $0.5 \mathrm{eV}$, and UV biophotonic energies measuring $5 \mathrm{eV}$, explaining how the brain may utilize an inordinate amount of energy without a corresponding increase in the presence of ATP. ${ }^{\dagger}$

\section{Blood biophotonic processes}

Superoxide production takes place in the neutrophil group of white blood cells in a way that differs from its generation in so-called respiratory bursts, or rapid consumption of oxygen resulting in its transformation into ROS (Voeikov et al., 1999). In a variety of ways, the ROS participate in molecular signaling processes. Whole blood in particular engages in an ongoing biophotonic process in which the ROS play a role that is physiologically essential yet unrelated to the immune function performed by the neutrophils (Voeikov, 2000, 2003b). One can observe something of a dance in the remarkable interplay of functions between red and white cells as the neutrophils (primary ROS source in blood) perform a transformative function on the oxygen transported by the erythrocytes (Voeikov, 2003b). Here the blood seems to act with conscious purpose as a whole system or organ rather than an aggregate of cells; it functions as a holistic unity. ${ }^{\dagger}$

Monitoring of spontaneous and luminophore-amplified photon emission (PE) from nondiluted human blood (i.e., under resting conditions as well as artificially induced immune reactions, see Fig. 1) reveals that blood is a continuous source of biophotons that store their energies in EES. This state is pumped by the generation of electron excitation produced in the ROS reactions. Excited states of blood and of neutrophil suspensions are oscillatory, suggesting interaction between individual sources of electron excitation. EES in blood are extremely sensitive to the tiniest fluctuations of external photonic fields but resistant to temperature variations as reflected in hysteresis of

\footnotetext{
$\dagger$ Voeikov VL. Conversations held in St. Petersburg, Russia, July 7, 2003.
} 


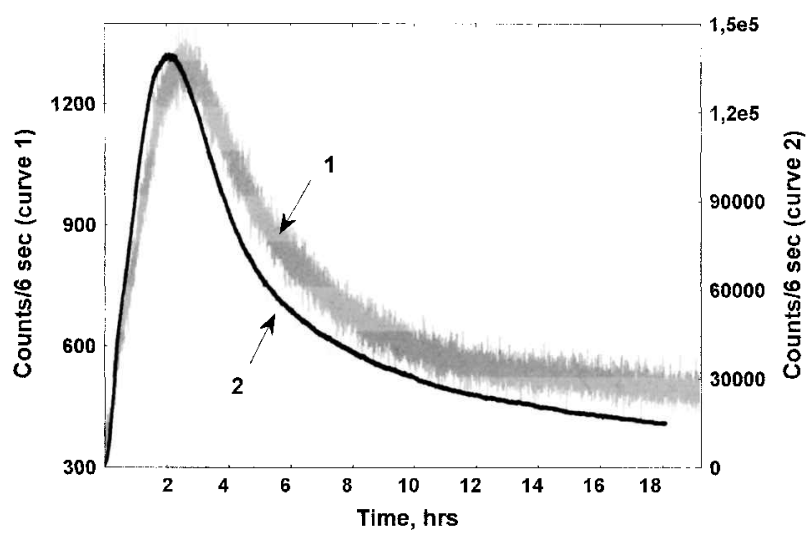

FIG. 1. Photon emission from nondiluted blood $(0.1 \mathrm{~mL})$ without luminol (curve 1, left Y-scale) or with it (curve 2, right Yscale) after induction of respiratory burst by addition of zymosan to blood (without zymosan and luminol photon emission [PE] from छblood sample was 250-300 counts per 6 second). Figure courtesy ğof V.L. Voeikov.

PE in response to temperature variations. These data sugSggest that blood is a highly cooperative nonequilibrium and Enonlinear system, whose components continuously interت्ञ act in time and space. At least in part this property is provided by the ability of blood to store energy of electron excitation that is produced in the course of its own nor.ू.mal metabolism. Further study of these qualities of blood may be able give us the basis for new diagnostic procedures (Voeikov, 1999).

In short, medical research can only benefit by exploring त़the implications of Voeikov's fundamental insight that: "the ipprocesses with ROS participation taking place in aqueous S.systems and generating electron excited states had played rand currently play fundamental bioenergetic and bioinformational role in the emergence and realization of vital „̋processes" (Voeikov, 2000).

On a pragmatic level, the reality of biophotons offers a uni-

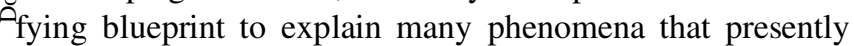
challenge the dominant biomedical view of life based on molecular reductionism. For example, it provides the rudiments of a scientific foundation for certain types of complementary and alternative interventions that involve the transfer of bioinformation carried by extremely small energy signals, such as those associated with the acupuncture feedback system, homeopathy, and quantum entanglement of information (Smith, pp. 69-78), electromedicine (Becker and Selden, 1985; Gerber, 2000; Liboff, pp. 41-47; Smith and Best, 1989), and spiritual healing involving the transmission and movement of energy (Rein, 2003, 2004; and this issue pp. 59-68). van Wijk has hypothesized $(1992 ; 2001)$ that these various circulatory systems may work by interacting directly with biophotonic emissions, making an impact on the global regulatory processes of life, rather than making an impact on the physical structure of the body (Popp, 2000). The next section highlights the com- plex information exchanges by means of coherent biophotonic fields, acoustic fields, and transmitted radio waves lying at the heart of the chromosomal "matter-wave function" and complex feedback sytems that govern bodily regulatory processes.

A biophotonic field is proposed as the complex organizing field of the organism comprised of well-known energy fields (electromagnetic, acoustic, radiologic) and more subtle fields of information involving non-local or universal consciousness not yet explained by science. The electromagnetic component of the biofield is seen (Van Wijk 1992, 2001, Zhang and Popp, et al., 1994) as a complex dynamic standing wave produced by the superimposition of all component waves of the electrically charged oscillators of the organism, ranging from the atomic, molecular and cellular levels to that of whole organs and organ systems including the heart and the brain. A closer examination of the molecular biology within the human body reveals its transmission abilities.

\section{THE BODY AS BIOCOMPUTER}

\section{DNA: Wave-biocomputer}

Research into morphogenetic fields from Gurwitsch to Sheldrake has reached a more refined state under the laboratory studies of Gariaev and associates working at the Institute of Control Science of the Russian Academy of Sciences. Here the structured processes generating these endogenous biofields that prefigure the formation of molecular structure are located within the chromosomal apparatus modeled as a laser emitter functioning with coherent (phase-aligned) biophotons. According to the Gariaev group (Gariaev et al., 2000), the chromosome functions as a global apparatus the fundamental property of which lies in the nonlocality of its genetic information. More specifically, the chromosomes work in terms of a quantum nonlocality of images expressed as indications of photons, namely a holographic image, which then supplies the information for the vectors of morphogenesis. In other words, chromosomes are the carriers of the order for our ultimate structure as an integral adult insofar as biologica "immortality" is passed from one individual to the next in creating the next individual in line.

It may be that the kinds and levels of thinking of which each of us is capable is essentially determined by the genes that are contained in each of us. It may be that each of our private languages is genetically determined. If this is true, that there is genetic scripting in regard to the way we think, we may have arrived at the point at which we can specify the levels of abstraction and the cognitional and theoretical parts of our composite body-brain-mind as entities that are genetically controlled. Conservative estimates suggest approximately $10^{14}$ operations per second in the human brain performed on $10^{21}$ molecules (Pauling, 1961). Thus, biologic form is prefigured in a quantum biohologram (Marcer 
and Schempp, 1998). Understanding the biocommunication and the quantum biocomputation at the heart of this process shows the living cell to be a biocomputer based on DNA. In this manner, DNA exhibits quasiconsciousness (Gariaev et al., 2000) in the context of the living cell where it is the script for what could be called the human biocomputer based on DNA (Gariaev, 1994) and where its possibility as a wave genome expands the principle of computer construction and satisfies the conditions specified by $\mathbf{J}$ von Neumann (von Neumann, 1966).

This model stipulates the basic double-helix structure to be two complementary or antiparallel strands as the necessary form to create a hologram that can reconstruct the whole, via a non-linear model of persistent wave-resonant holographic form that allows recognition of antiparallel half strands. Thus, the chromosome system acts as both sender and receiver of the genetic "texts" wherein its holographic omemory is read by electromagnetic and acoustical fields that F carry the gene-wave information out beyond the limits of ợ the chromosome itself. This activity exhibits at the level of the chromosome itself, the matter-wave duality of the non1ocal signal processing. Such behavior may indeed be analJogous to what Pribram (1991) has theorized for the memSory process within the cerebral cortex. But is it quantum Finformation?

Gariaev and coworkers maintained that theory predicts a Ematerial apparatus of the chromosome coupled with fast. optical properties, such that the chromosomes of the separate cells of an organism are actually connected as a holistic scontinuum producing laser radiations as one of their own ज़̣field types. This is just what their experiments have veriत़fied. Working with in vitro DNA preparations, it was found that the DNA would interplay with a special two-beam laser, in this case $\lambda=632.8 \mathrm{~nm}$, changing the polarization of the ज्ञight and converting it simultaneously into a radiofrequency 帝range. Remarkably, the photons were modulated by molecoles of the DNA preparation. In this manner, the re-

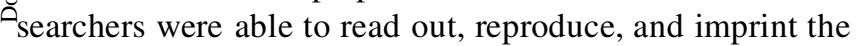
stored DNA information. Moreover, the DNA-generated radio waves appeared as an entirely new type of signal, in which information is encoded by polarization of electromagnetic vectors (Gariaev et al., 2000).

Application of this experimental methodology has resulted in the observation of several extraordinary phenomena, most notably the use of laser-generated DNA information to resuscitate damaged Arabidopsis thaliana seeds that had been irradiated at Chernobyl. Polarized radio waves not carrying the DNA information failed to resuscitate the damaged seeds. Additionally, DNA-generated radio waves exhibited their high genetic activity by producing superfast growth in potatoes of up to $1 \mathrm{~cm}$ per day, along with tuber formation on stalks as well as roots (Gariaev et al., 1994). Once developed, this technology could be applied medically to repair damaged chromosomes in a noninvasive manner, and to tackle incurable diseases such as human immunodeficiency virus (HIV).

\section{DNA fluctuation dynamics and the quantum biocomputing process}

What we are looking at is a wave theory of chromosomal functioning in which the fluctuation dynamics of the genome read, code, and transmit the information from one part of the body to another, beyond local (on a molecular scale) distances (Gariaev et al., 1994). By virtue of its dynamic ability to read information throughout the body as part of its nonlocal quality, it can read and alter its own holographic information field as an oscillator-generating quantum holography by means of a biosolitonic process model. The soliton, as ultrastable wave train, is much like a quantum exhibiting a wave/particle duality. It has the capacity to convey information. Solitonic processing in DNA is critical to quantum computing in terms of the reading of the codons. By means of their experiments the Gariaev group arrived at the conclusion that: "DNA functions as a quantum coherent system/assembly (of now quantum oscillators) or whole, by means of quantum entanglement" (Gariaev et al., 1994). The polarization of the holographic images by means of a completely new electromagnetic vector substantiates the case for the DNA molecule being an actual quantum mechanical harmonic oscillator functioning with nonlinear oscillations.

Clearly, the implications of this for the future of medicine are far-reaching and bordering on the fantastic. Already, we can see the possibility of "wave vaccines," which according to Gariaev and colleagues will only require a broader spectrum laser that can function over the full color regions within which the genome operates (Gariaev et al., 2000). Such lasers could imitate the wave signature function of chromosomes and transmit wave genetic information between two genomes, thus making it possible to control metabolism. This, in turn, would enable the correction of genetic defects, the restoration of damaged chromosomes (as already demonstrated with the seeds from Chernobyl), and new approaches to cancer and HIV in mechanisms and therapies.

What might this bioplasmic continuum tell us about the mystery of our own nature? The liquid crystal nature of DNA exhibits a plasma-like activity that appears to be a unified whole, or holon. In some ways it exhibits a consciousness that functions as a biocomputer that operates with incredible thoughtfulness beyond our cognition of it. Its hyperkinetic nature of rapid vibratory activity and quantum computation may provide a theoretical understanding to enable the design of future inventions such as quantum computers and new holographic video technologies (Gariaev et al., 1994), but it is even more promising in terms of understanding our pluriform nature. The instant media and nonlocal activity of our wave-genome biocomputer seems to form what might be termed an "epikinetic body," preceding 
action potentials and prefiguring material formation. In that regard it represents information that may be a transduction of even subtler fields. Sheldrake (1988) referred to these as morphic fields; "non-material regions of influence." For our purposes it adds to the groundwork for a Multi-Bodied Transduction Model (MBTM) that can make sense of the human being as a complex of interpenetrating consciousness and energy fields as vibratory bodies (Hurtak, 1973b).

\section{ACUPUNCTURE: CIRCULATORY SYSTEM FOR THE ELECTROMAGNETIC BODY}

\section{Acupuncture and high-speed communication}

Evidence of a hyperfast communication system in the body seems to indicate that the human body works as a bioơcomputer, operating on many levels of information simultheously. In the previous section we examined the oscilô thergetic change from the quantum to the atomic to the com$\stackrel{\sim}{m}$ plex molecular. In another domain, studies at the University đof California, Irvine, have demonstrated the transmission of Finformation at speeds of several orders of magnitude greater than nerve impulses (Cho et al., 1998). This was the first ¿study to demonstrate a direct correlation between stimulation of acupuncture points and brain activity. Stimulation of 敋he visual cortex of the brain was compared between flashes of light and more remote activation of specific acupuncture points in the foot. fMRI and ultrasonic imaging revealed increases in cortical blood flow within the regions of the viIsual cortex with stimulation of acupoint BL-67 on a blindifolded subject comparable to that achieved through direct stimulation by light to the eyes. The lighting up of the brain took place within only a few hundred microseconds while ơdirect visual stimulation required approximately 180-200 ŏ milliseconds (ms) to reach the brain through nerve impulse. Discussion of this hyperfast communication within the body can be found in the abstract of a presentation by Jones et al. (2002) given at the 21st Society for Scientific Exploration (SSE) conference. While it may not be at quite the velocity of an e-m pulse, it is more than 2-orders of magnitude faster than the nervous system. We could be witnessing something like a fifth circulatory system (Hurtak, 1973a).

Support for this distinct communication network lies in the point-specific activation of the brain response, to wit the response only occurred with stimulation of the acupuncture point. Moving off of the point produced only noise. Furthermore, Jones et al. used ultrasound to sense the energetic flow that they equated with the movement of $q i$ and found to move at approximately a few centimeters per second. Additional activity in the visual cortex associated with visualrelated acupoints confirmed the velocity by the time interval until cortical response. Sensitive individuals likewise noted a flow of energy from the acupoint moving along the meridian at a slow rate that further corroborates such an energetic flow. This confirms at least the possibility of multiple pathways at work (Jones, et al., 2002), and here we see three: (1) the hyperfast communication between acupoint and brain measured at no more than $0.8 \mathrm{~ms}$; (2) nerve impulse along nerve pathways registering an interval of 180-200 ms until cortical response, and (3) slow moving energy flow associated with $q i$.

Jones and $\mathrm{Bae}^{\ddagger}$ also used ultrasonic visualization to image acupoints, discovering that given acupoints such as BL67, BL-66, BL-65, and BL-60, changed in size, shape, and even location, when observed over a 12-day period. Furthermore, the points were found to represent regions of enhanced ultrasonic attenuation thus susceptible to accurate ultrasonic mapping and stimulation as well. Because all of the acupoints imaged in this study appeared to be located within the connective tissue, an observation in agreement with findings of Langevin and Yandow (2002), we must be willing to consider alternatives to an anatomic structure serving as a conductive system. In other words, while the jing luo may interpenetrate certain spaces within the body (Ma et al., 2003), it operates on a different energetic spectrum, thus passing through a common space, utilizing the molecular structure, not like a tube or vessel, but rather like an energetic wave moving through commonly bound clouds of electrons. The discovery of these new interpenetrating lines suggests a new level for the functional organization of the human as a biocomputer.

Indeed, this is our position, and we find it well supported in the work of Korotkov et al. (Korotkov, 2002). As with the Jones group, channels of conductance are located in the mass of connective tissue. There, formations of particular structural-protein complexes serve as channels of increased electron conductivity, an insight that is supported experimentally by the measurement of surface electrical conductance at acupuncture points (Syldona and Rein, 1999). In short, the Korotkov team has associated the Eastern idea of "energy" transfer, with "the transport of electron-excited states through molecular protein complexes" (Korotkov et al., pp. 49-57). In effect, the organism is creating an energy store in EES available for deployment when needed.

Movement of electrons and/or wave trains through connective tissue remains consistent with other studies done by Zhang et al. (2002). For example, skin conductivity, a property that has some correlation with the acupuncture system reveals that both acupuncture points and meridians are not fixed, but part of a dynamic system that can exhibit wide variance due to physiological or psychological pathology. Dynamic behavior of this type could well occur via molecular ensembles of proteins conducting e-m waves where no fixed structural conductors exist.

$¥$ Jones JP, Bae YK. Ultrasonic visualization and stimulation of classical oriental acupuncture points, unpublished paper. 
Research done in China has shown that acupuncture meridians are also good channels for both electromagnetic and acoustic waves. Chinese physicist B.W. Zhang found acumeridians to be excellent conductors of microwaves, in particular at the frequency $1359 \mathrm{MHz}(\mathrm{Hu}, 1990$; Zhang, 1961). He further postulated that the acumeridian functions as a type of "wave guide" in which the slow signal speed comes from the "group speed of waves." This may be the best explanation to date for the slower energy movement similar to that reported by Jones et al. Zhang's application of "group waves" in 1959 really predated recognition by the scientific community of dissipative structure. Use of soft-laser acupuncture by medical doctors in Western countries further demonstrates the light conductive capacity of the meridians. Thus, acupuncture meridians serve as channels of electromagnetic waves, 저hether in the visible light range or in the microwave region of the e-m spectrum. Chinese physicist P.S. Sun and Oothers (Hu et al., 1990; Sun, 1988) have found a high deơgree of similarity and coincidence between higher sound Fintensity points and meridians on the human body. This result supports the hypothesis that the meridians also conปdduct acoustical waves.

\section{EAcupuncture and the fifth circulatory system}

Existence of the acupuncture system as a completely dis. ther confirmed by experiments that seek to address the qquestion of whether the acupuncture system exists or not Eafter death. Chinese biophysicist Z.X. Zhu (1989) observed त़the lower resistance points and the higher sound intensity ipoints after amputation. Results show that the lower resisStance points remain almost exactly in their original locations after amputation. Therefore, researchers will need to Dinvestigate the acupuncture system as one that is a comofpletely distinct energy circulatory system interacting with the biomolecular structures but surviving their dissolution for some time.

In point of fact, the interference pattern of electromagnetic waves inside the human body is a dissipative structure, which is relatively stable as long as the energy support in the body is present (Hurtak 1971). On the other hand, it is a very dynamic structure which accounts for the large fluctuations in various frequencies observed with highly sensitive instruments. Changes in the boundary condition of the resonance cavity, or energy support, corresponds with pathological changes in physiology and psychology leading to alterations in the contours of the interference pattern. This, in turn, would explain the moving profile of acupoints and meridians under nonordinary psychologic and physiologic conditions (Zhang, 2002).

Now, it is well documented that the holographic phenomenon is a basic characteristic of any interference pattern, as the Gariaev group observed with the genome. Analogously, the holographic dimension of the acupuncture system as circuits of energy in the body is no longer a mystery but can be seen in terms of its interference pattern, producible not only by electromagnetic waves, but also by acoustic waves that also give rise to interference patterns. Action of the interference pattern makes diverse phenomena much more intelligible. For example, the slow speed of "sensation propagation" (Jones et al., 2002) or the change of so called "skin resistance" can easily be explained by the "group speed" of waves along the wave guide, which is a part of interference pattern. It has been noted that such patterns persist in a dead body, and in other biologic bodies such as fruits and plants provided they are resonance cavities with continual energy support. (Note: the measurement current itself may be a source of energy support). Finally, from the perspective of holographic interference patterns, we would have a completely new understanding of the mechanism at work in acupuncture needling. Thus, a metal needle functions as a new boundary condition that can be introduced into a body in such a way as to alter its interference patterns. Rotation of the needle can cause attachment of connective tissue fibers. Provided needling is done in an appropriate location, it can bring about a great change in the interference pattern in the direction of more harmonious global regulatory activity. The decisive place is most frequently at the point of highest electromagnetic field strength, at the confluence of many peaks of electromagnetic standing waves (Zhang, 2002).

Indeed, while the Western confrontation with the phenomenon of acupuncture has prompted ingenious attempts to locate a physical basis for the jing luo or meridian system, these have all failed to find a definitive correlate for the information or energy flow. A very interesting recent hypothesis (Hu et al., 2003) asserts the locus of the jing luo within the perivascular space as a possible substrate. Even if this might turn out to be a valid location, it has not been demonstrated to be more than a space interpenetrated by the energetic flows. A more compelling critique of anatomical structure as conveyance mechanism lies in the shifting location and footprint of both the meridians and the points. This phenomenon suggests rather an energetic system and possible alternative body along the lines of what C.L. Zhang $(1996,2002)$ has suggested as part of a dynamic dissipative structure that is a standing electromagnetic wave in and around the physical body. Nobel Prize-winning Belgian scientist Ilya Prigogine introduced this concept of "dissipative structure" within a context of open systems rather than closed systems, and under a nonlinear thermodynamics operating far from equilibrium (Prigogine and Gregoire, 1977). This is foundational to the current understanding of the human system as homeodynamic rather than homeostatic. Beyond this, however, the dissipative structure needs to be understood in terms of coherence (Zhang et al., 1994; Zhang, 2000). The log-normal distribution of skin-conductivity values shown in Figure 2 further confirms the dissipative structure of this electromagnetic standing wave "body." 


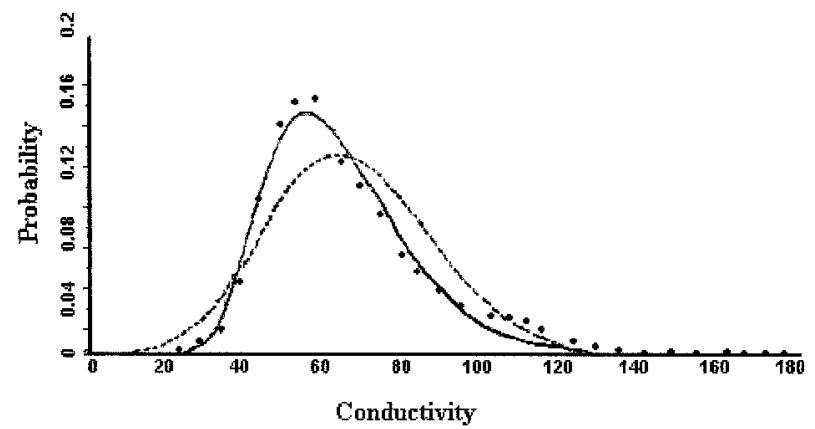

FIG. 2. The probability distribution of 18,000 skin-conductivity values of 200 healthy people follows a log-normal distribution (continuous line) instead of a normal one (Zhang, 1994). Figure courtesy of C.L. Zhang.

Syldona and Rein (1999) have likewise postulated the acupuncture meridian system as an underlying template for othe physical body. Liboff (pp. 41-47) further makes a conJvincing case for an electromagnetic paradigm for the human obeing. Science may soon come to recognize a distinct, yet therconnected body that we may term the electromagnetic body (Zhang, 2003). Such a realization would amount to a İmacroscopic instance of what Walach et al. (2000) have termed "complementarity," namely, the biophysical expresEsion of the quantum mechanical principle, where both wave and particle natures are manifested. The Uncertainty Prinô. ciple still obtains between the number of particles and their ophase.

In view of the electromagnetic and acoustical holographic genergy/information forms present within the genome we are Enaturally drawn to examine the parallel case within the İmeridian grid system. Is this a possible point of consonance Tand therefore a gateway of interaction between the bioplas-mic "body" holism formed by the continuum of the genome "zand the electromagnetic standing wave "body" within and Earound the chemical and molecular form? Only further labбू̆ ddence to highlight the interaction of the two systems as part of a larger light continuum.

Nevertheless, the authors are convinced by the results before us to consider that the human body functions at some levels as a quasi-“light" body transducing signals locally and nonlocally, both from within itself and from extrabiologic sources (Gariaev et al., 1994; Hurtak, 1996a). Difficulty in reproducing the Gariaev experiments is because of the complex dual-laser system utilized, and renders the theory speculative. The current advantage of the theory lies in its ability to provide a unified explanation that encompasses diverse levels of energy transduction within one holistic system.

\section{The astro-acupuncture system and medical astronomy}

Thus, in our view, acupuncture works with a fifth circulatory system of light (biophotons) connected to an internal current that operates through the acupuncture meridians.
This light circulatory system is constantly working, able to shape the action potential of the total body system. In the body this gives rise to a substratum of direct current potentials as another mechanism of data transmission. These preexisting direct current (DC) potentials, in turn, behave as governing functions that control the basic properties of living organisms (Hurtak, 1973a). This model can account for the ability of more subtle light signals to interact with physical regulatory functions via the acupuncture/electromagnetic biosystem.

Viewing the universe as a fractal totality, the human body appears as a micro-cosmos, a small space-time field within a larger field. In fact, evidence of a global morphological factor nested within the harmonics of a global standing gravitational wave substantiates this claim (Müller and Granögger, 2001). From this perspective acupuncture stands out as the first empirical demonstration of systematic field behavior and the physical-physiologic interaction of two universes. The interaction takes place by means of a new physiologic system that functions alongside the accepted blood circulatory, lymphatic, and nervous systems-the electromagnetic (Hurtak, 1973a, 1996a, 1996b).

Precisely because of the dramatic discoveries of the Gariaev group, and Zhang and colleagues we must now seriously investigate this interaction of the matrix of interpenetrating fields in the body with other distant and subtle information fields emanating from other nodal points of consciousness in the cosmos. This idea is not entirely novel, as contemporary researchers in China have pointed out the larger acupuncture system that was once viewed as extending beyond the limits of the human body and into the greater cosmos (Zhang, 1961; Hurtak, 1973a, 1988). The greater astroacupuncture system allows induction of bioluminescent and electrical signals using fractal networks to override normal cause and effect (Hurtak, 1988). It is beginning to appear that humankind is working only with a truncated version of acupuncture when compared with the ancient practice. Moreover, the newest translations of the Nei Jing (Book Review by Pham; pp. 191-196) may confirm this more expanded model of acupuncture science.

Therefore, we should begin to examine our human acupuncture system even more holistically, as an extended astro-bioacupuncture system that is, in degrees of complexity, connected to communication circuits with the various internal and external meridians as depicted in ancient Chinese texts (Fig. 3). This extended system could be called an axiatonal system of vibratory communication, by virtue of its functioning through meridians with diverse fields of light and sound (Hurtak, 1973a), a macrocosmic ordering of what Gariaev et al. (1994) are observing in the acoustico-optical properties (i.e., the frequency properties) of the genome. We see on the basis of extrapolation of current research in new energy medicine, the emergence of a whole new branch of medical science that can be called "Medical Astronomy." Vedic sages were schooled in an analogous science known as Jyotish thousands of years ago in India. Through research 


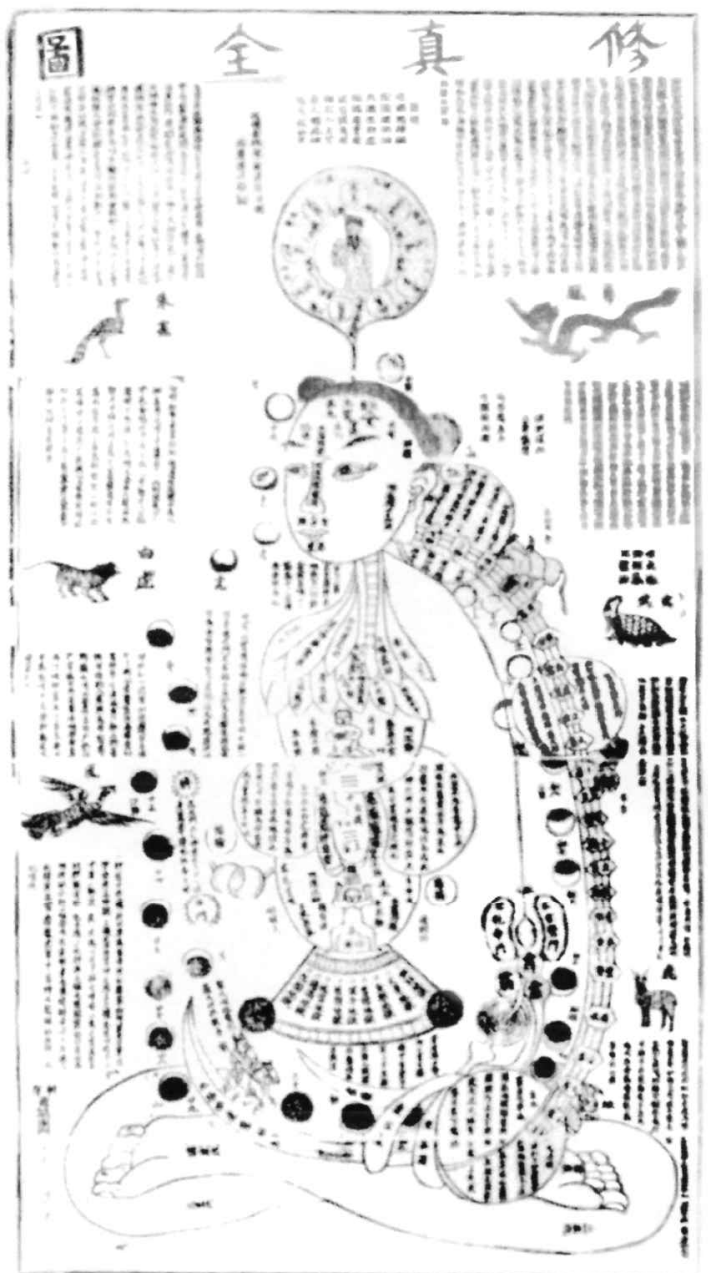

TFIG. 3. Acupuncture chart depicting the human system as a त̣macrocosmic mind-body relationship. Relations are portrayed as Sincluding both transpersonal self and astronomical alignments. Figजُure courtesy of J.J. Hurtak.

㻤.

ซ్ in medical astronomy we can deepen humanity's underEstanding of these bioluminescence and electrical impulses ๑connected with the origin of cell division, so that "modern medical techniques should soon be able to tap into the nodal of cell memory and stimulate it in order to develop new limbs, organs and tissues. Thus we can have the rebuilding of the human body cell-by-cell" (Hurtak, 1973, 1996).

\section{CONSCIOUSNESS, QUANTUM ENTANGLEMENT, AND THE MEDICINE OF LIGHT}

\section{Mind-body-energy research}

The research of Pribram (1969, 1977, 1991), Gariaev (1994), Stapp $(1988,1993)$ and others involved with important breakthroughs in basic neurophysiologic and molecular biologic research of human consciousness, has opened the way for new models of mind/body relations as well as expanding the conception of mind-brain interaction. von Neumann, however, had rigorously formalized the importance of mind in all quantum considerations. New studies show further that consciousness works beyond the cellular and molecular levels and may guide us to understand the ultimate mystery of nature, namely, how consciousness works as a multidimensional matrix of energy fields and correlated event related signals between distant human brains as manifestation of the non-locality of quantum systems (Crawford et al., 2003; Radin et al., pp. 103-112; Stapp, 1988; Tiller et al., pp. 145-157; Wackerman et al., 2003).

One exciting line of investigation involves extended network theory and macroscopic quantum interactions known as entanglement (Hyland, 2003), similar to the microscopic quantum entanglement known by the Einstein-PodolskyRosen paradox-here particles behave as if in communication with each other when their spatial separation disallows that possibility (Aczel, 2003). Entanglement and actual communication between entangled entities requires non-linear quantum theories (such as might account for the nonlinear oscillations of DNA); linear-based theories cannot support nonlocal instantaneous information transfer between quantum entities. Such phenomena appear as a large-scale version of the quantum entanglement evident within the DNA harmonic oscillator. It is worth noting that we must delineate between quantum and (spatiotemporal) biofields; e-m explanations of healing phenomena and a-temporal, supraspacial entanglement (L. Milgrom, personal communication, September 3, 2003). The upshot for medical research will be a light medicine that goes beyond electromagnetics (Rein, pp. 59-68).

For low-frequency electromagnetic fields one fundamental problem involves the dynamic by which weak ambient infrasonic waves interact with the body, which clearly must be coherent down to the frequencies of circadian rhythms. It is known that the human ear, unless assisted by electronic amplifiers, cannot respond to infrasound because the work of European investigators such as Von Bekesy and others calculated the threshold level at pressures of 2000 dyne $/ \mathrm{cm}^{2}$ at $1 \mathrm{~Hz}$ Hertz. However, the human body vibrates between 4 and $10 \mathrm{~Hz}$ with amplitudes of $10 \mu \mathrm{m}$; hence, under muscle tension and relaxation the physiologic functions of the lungs and joints and bones of the body can be influenced directly by certain combinations of sound and music vibratory levels through which the human body becomes a musical instrument.

In the early 1970 s, noted inventor of the video synthesizer, Nam Jun Paik, electronic music legend Morton Subotnick, who worked at the Sorbonne, and J.J. Hurtak Ph.D. (Social Science), Ph.D. (History \& Oriental Studies), carried out biofeedback studies with their students at California Institute of the Arts, Valencia, CA, showing that the circuits of the body at the chakra points could be controlled through music and biologic linkages with meta-signals from higher states of consciousness. A series of metaprograms of mantras were combined with musical signals that were then 
creatively expressed on a video screen and enhanced through a biofeedback "interlock" with geometries on the screen. Through this process, a micro-picture of patterns of mental signals, energy-force-material exchanges with outside sources, could be produced that showed the human body as a musical transducer for which repeatability, reliability, and signal/noise relations could be established. With many of the students, an integrated-over-time picture of repeating musical elements appeared as extended flashes on cue on the screen (that is, in a series of milliseconds to a microsecond of a frozen picture or patterns). This demonstrated the power of biofeedback on a "meta-level" of consciousness orchestrating patterns and music simultaneously.

Although no scientific paper was published, a series of sound recordings were created with important contributions from frequencies between $1 \mathrm{~Hz}$ to $10 \mathrm{~Hz}$ as a result of the biocoupling between meta-images and the unique video imoges that would appear on screen. Use of this type of metabiofeedback later eventuated into an award-winning comôّ Tentrainment of the mind, or a many-body approach to a con"sciousness reality resulting in "the Buddha." It is clear to the authors, partly on the basis of all of the aforementioned Jresearch, that there exists within everyone, on a supraphysFical level, a consciousness body of tremendous holographic oflanguage potential. Through the external changes in the human body, images can be reflected in internal changes

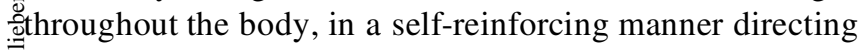
the release of "shakti" or psychophysical signals from the mind into all major body circuits through which the sense हैof well-being and happiness is enhanced. Musically induced त्र̣biocomputer waves can cross boundary structures between त़the different biochemical and sensory bodies (blood vessels, Inerve fibers, cerebral spinal fluid) resulting in a unified cirincuitry which may be the foundation for electro-medicine.

of

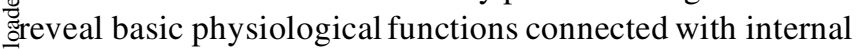
organs from a new perspective in the development of elec-

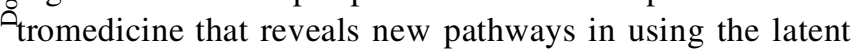
energies of our body. These achievements will come through a combination of understanding the electromagnetic-bioluminescence of the body and the Chi pattern underlying the acupuncture system" (Hurtak 1973a, 1996b).

\section{The many-body approach and the continuum of consciousness vehicles}

In summary, studies of human consciousness are important in showing the structures of multibody vehicles that already exist within the complex of the human being as a living holistic combination of fields that exist as a microuniverse in the body (Fig. 4).

As humankind discovers its capacity as a biotransducer for multiple energy fields from the cell to the quantum mind, breathtaking discoveries will show the true interconnection

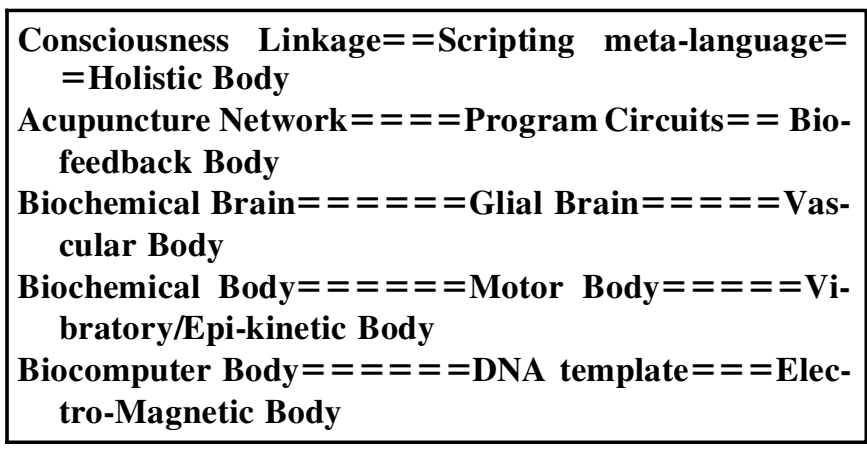

FIG. 4. Multibody transduction model showing various circulatory systems as consciousness transducers. Figure courtesy of J.J. Hurtak Ph.D. (Social Science), Ph.D. (History \& Oriental Studies), 1973, for the Academy for Parapsychology and Medicine.

of bio-transmission systems and communication channels in the living lightware that makes life the connection between the human and the divine. The profound impact of the biocomputer is the next chapter of the human evolution unfolding into the higher evolution. In the near future there will be an increased acceptance of electromedicine, medicine that operates upon the foundations of the biocomputer networking of light, that "controls the spatial quantization necessary for the balance of the body in relationship to the medula, pons, midbrain, thalamus, and hypothalamus, which can be stimulated to accept the new limb, organ or tissue" (Hurtak, 1977).

Holographic organization behind the seeding of superholograms from a greater template is consonant with the foundational modeling of Pribram (1991), and the expanded theoretical framework of Gariaev (1994). "Holographic organization shifts emphasis from axonal impulses to the slow potential micro-structure that develops in the post-synaptic networks . . . In man, given the neural hologram, these steps lead to 'image constructions' of light" (Hurtak, 1977).

We will need to use quantum mind studies of consciousness and the physical sciences from biology to physics to define the medicine of light broadly. As a constellation of healing therapies it will include photonic applications and electro-medicine, now moving well beyond its early foundations (Becker and Selden, 1985; Liboff, pp. 41-47). Beyond that, greater promise may lie in the exploration of wave genomics where information is coded and transmitted to the chromosome apparatus in the form of a "wave vaccine" as the Gariaev group has suggested. Application of DNA-waves (either nonlocal or "injected"), in conjunction with molecular biology (when this dual communication link is regained through the modulation of DNA), will allow tRNA molecules to cause cells to alter their normal properties to receive the original genetic transmissions given through a spin point to a cell. A spin point is a connecting point between the fifth or light 
circulatory system and the nodal points of cell memory. These transmissions at the spin points change the spin of cell molecules, enabling regenerating instructions for the manufacture of enzymes and proteins, the building blocks for the new tissue, or new organ that is regenerated on the physical plane (Hurtak, 1973a).

On this furthest horizon of medical science we look to the continuum of humanity's consciousness vehicles, perhaps harnessing the power of the ancient science known as the Vedas (the wisdom and knowledge of the ancient Rishis of India) where one may participate with the five Koshas or consciousness vehicles that can function as a life/light continuum, connecting humankind to more subtle realms of existence; more advanced consciousness time zones. In contemporary terms, we may refer to this consciousness continuum as MBTM, analogous to the wisdom of the Vedas that speaks of the five Koshas or five consciousness vehiog cles. Exploration of a verifiable MBTM will become necEessary to understand how light is transduced in the human

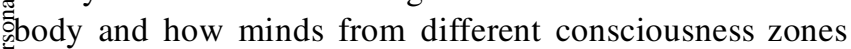
Smay interact with one another. Understanding the chakras, the acupuncture meridians, and the quantum coherence of Itthe body is just a part of the story. Without the continued Einvestigation of a supraphysical consciousness body of tremendous holographic language potential, our healing therapies will be working with an incomplete map of the :

In this review of the frontier medical science of our age, we have observed evidences of several of the consciousness vehicles referred to in the Sanskrit tradition, including the ¿्.namaya Kosha, or "electromagnetic body" and the Pranaत̦maya Kosha, or "epikinetic body" of vibratory harmonics तileading to higher states of the biophysical. Will the mapping Sand experiential expansion of these "bodies" open up a ingreater Medicine of Light? Can we look to the promise of zaccessing a divine blueprint, the nonlocal template containज्ञng the primordial subtle fields that provide the ontogenesis of morphogenetic fields in the quantum realm? This vision

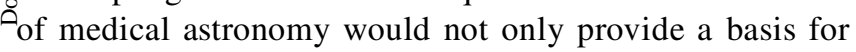
unified field theory, but usher in a wonderfully whole era of light in which the fullness of health would be the birthright of each and every person. To that end we, as a planetary society, must attend to the careful and rigorous investigation that will one day engender the fullness of this emerging body of medical science that truly could be called the Medicine of Light.

\section{ACKNOWLEDGMENTS}

This work is the product of many dedicated minds diligently pursuing the deeper truths of our physical nature, and its relation to our consciousness. In particular, we would like to thank Konstantin Korotkov and Vladimir Voeikov for the lengthy and profound discussions that added so much substance in illuminating the life foundation of biologic systems, and contours for a new paradigm in the biologic sciences.

\section{REFERENCES}

Aczel AD. Entanglement. Chichester, UK: Wiley, 2003.

Becker RO, Marino AA. Electromagnetism and Life. Albany, NY: State University of New York Press, 1982.

Becker RO, Selden G. The Body Electric. New York: William Morrow, 1985.

Bischof M. Some remarks on the history of biophysics and its future. In: The Current Development of Biophysics. Hangzhou: Hangzhou University Press, 1996:10-21.

Bischof M. Field concepts and the emergence of a holistic biophysics. In Biophotons and Coherent Systems, Beloussov L, Popp FA, Voeikov V, van Wijk R, eds. Proceedings of the 2nd Alexander Gurwitsch Conference. Moscow: Moscow University Press, 2000.

Beloussov LV, Popp FA, eds. Biophotonics: Non-equilibrium and Coherent Systems in Biology, Biophysics and Biotechnology. Moscow: Bioinform Services Co., 1995.

Bohm D. Wholeness and the Implicate Order. London: Routledge, 1980.

Chang JJ, Liu Y, Wang Y, Popp FA. Biocommunication and Bioluminescence of Lampyridae, in Biophotons and Coherent Systems, Proceedings of the 2nd Alexander Gurwitsch Conference. Moscow: Moscow University Press, 2000.

Cho ZH, Chung SC, Jones JP, Park JB, Lee HJ, Wong EK, Min BI. New findings of the correlation between acupoints and corresponding brain cortices using functional MRI. Proc Natl Acad Sci USA 1998;95:2670-2673.

Crawford CC, Jonas WB, Nelson R, Wirkus M, Wirkus M. Alterations in random event measures associated with a healing practice. J Altern Complement Med 2003;3:345-353.

Dürr HP. Presented at Gelterswoog Symposium on Biophysics, March 14, 1998. Kaiserslautern, Germany, 1998.

Gariaev P. Wave Genome [in Russian]. Moscow: Obchestv Polza, 1994.

Gariaev P, Birshtein BI, Iarochenko, AM, Marcer PJ, Tertishny GG, Leonova KA, Kaempf U. The DNA-wave biocomputer. Moscow: MGU, 1994. Presented at Liege Belgium, 2000.

Gariaev PP, Tertishny GG, Leonova KA. The wave, probabilistic and linguistic representations of cancer and HIV. J Nonlocality Remote Mental Interactions 2000;1(2) Online document at: www.emergentmind.org/gariaevI2.htm

Gerber R. Vibrational Medicine for the 21st Century. New York: Eagle Brook, 2000.

Gurwitsch AG. The Theory of the Biological Field [in Russian]. Moscow: Sovetskaya Nauka Publishing House, 1944.

Gurwitsch AG, Gurwitsch LD. Introduction into the Doctrine of Mitogenetic Radiation. Moscow: USSR Academy of Medical Sciences Publishing House, 1948.

Hu XL, et al., eds. Modern Scientific Research in Acupuncture Channels and Collaterals in Traditional Chinese Medicine [in Chinese], Beijing: People's Hygiene Publishing House, 1990.

Hurtak JJ. The meaning of acupuncture for modern medicine. Town Hall Reporter Magazine, June 1971:13-15. 
Hurtak JJ. The Book of Knowledge: The Keys of Enoch. Los Gatos, CA: The Academy for Future Science, 1973a.

Hurtak JJ. Electromedicine and the fourth state of matter. Presented at the Academy of Parapsychology and Medicine [proceedings on audiotape]. Los Altos, CA, 1973 b.

Hurtak JJ. The human body-its energy and resonance matrix. Future History 1996a;2:1:7-10.

Hurtak JJ. Electrobioluminescence, laser radiation and acupuncture points. Future History $1996 b ; 2: 1: 11-14$.

Hyland M. Extended network generalized entanglement theory: Therapeutic mechanisms, empirical predictions and investigations. J Altern Complement Med 2003;6:919-936.

Jones JP, Wilson L, Bae YK, So CS, Kidney DD. Characterization of acupuncture points using fMRI and quantitative ultrasonic methods. Charlottesville: University of Virginia, Proceedings of the 21st SSE Conference, May 29-31, 2002.

Kasnacheyev VP, Mikhailova LP. Distant intercellularinteractions in a system of two tissue cultures. Psychoenergetic Syst 1976; 玄 $1: 3-15$.

\&Kasnacheyev VP, Mikhailova LP. The Role of Ultra-Weak Emissions in Intercellular Communication. Novosibirsk: Nauka, 1981 .

Korotkov KG. Human Energy Field-Study with GDV Bioelec-

trography. Fairlawn, NJ: Backbone Publishing, 2002.

SLLangevin HM, Yandow JA. Relationship of acupuncture points and

meridians to connective tissue planes. Anat Rec 2002;269: 257-265.

ठั้Liboff AR. Bioelectromagnetic fields and acupuncture. J Altern Complement Med 1997;3:S77-S87.

Ma W, Tong H, Xu W, Hu J, Liu N, Li H, Cao L. Perivascular space: Possible anatomical substrate for the meridian. J Altern Complement Med 2003;6:851-859.

Marcer P, Schempp W. The brain as a conscious system. Int J General Systems 1998;27:231-248.

స్Müller H, Granögger U. The universe's intrinsic oscillations: tri$\stackrel{\overrightarrow{7}}{ }$ nary structures in all natural processes. Future History 2001;3: 2-5,11-14.

ज̛ंMusès C. Working with the hypernumberidea. In: Musès C, Young

Aे AM, eds. Consciousness and Reality: The Human Pivot Point.

New York: Outerbridge \& Lazarus, 1972;448-469.

Pauling L. A molecular theory of general anesthesia. Science 1961;134:15021.

Popp FA. Photon storage in biological systems. In: Popp FA, Becker G, Konig HL, Peschka W, eds. Electromagnetic Bio-Information. Munich-Baltimore: Urban \& Schwarzenberg, 1979.

Popp FA, Li KH, Gu Q, eds. Recent Advances in Biophoton Research and its Applications. Singapore: World Scientific, 1992.

Popp FA. Some features of biophotons and their interpretation in terms of coherent states, in Biophotons and Coherent Systems, Proceedings of the 2nd Alexander Gurwitsch Conference. Moscow: Moscow University Press, 2000.

Pribram KH. The Neurophysiology of remembering. Sci Am 1969;220:75.

Pribram KH. Languages of the Brain. Monterey, CA: Wadsworth Publishing, 1977.

Pribram KH. Brain and Perception: Holonomy and Structure in Figural Processing. New Jersey: Lawrence Erlbaum Associates, 1991.

Prigogine I. Introduction to the Thermodynamics of Irreversible Processes. 3rd ed. New York: John Wiley \& Sons, 1967.
Prigogine I, Gregoire N. Self-Organization in Nonequilibrium Systems: From Dissipative Structures to Order through Fluctuation. New York: John Wiley \& Sons, 1977.

Radin D. Experimental investigation of event-related EEG correlations between isolated human subjects. J Altern Complement Med 2004 (in press).

Sheldrake R. The Presence of the Past: Morphic Resonance and the Habits of Nature. New York: Vintage Books, a division of Random House, 1988.

Smith CW, Best S. Electromagnetic Man. London: J.M. Dent, 1989, 1990; New York: St. Martin's Press, 1989.

Stapp HP. Quantum theory and the physicist's conception of nature: philosophicalimplications of Bell's theorem. In: Kitchener RF, ed. The World View of Contemporary Physics: Does it Need a New Metaphysics? Albany, NY: State University of New York Press, 1988:38-58.

Stapp HP. Mind, Matter and Quantum Mechanics. Berlin: Springer-Verlag, 1993.

Sun PS, Zhao YH, Li YL, Yan QL, Liu H. The study on conduction of acoustic information along meridians [in Chinese]. Chen Tzu Yen Chiu 1988;13:139-143.

Syldona M, Rein G. The use of DC electrodermal potential measurements and healer's felt sense to assess the energetic nature of qi. J Altern Complement Med 1999;4:329-347.

Szent-Györgi A. Towards a new biochemistry. Science 1941;93: 609.

Szent-Györgi A. Introduction to a Submolecular Biology. New York: Academic Press, 1960.

Van Wijk R, van Aken JH. Photon emission in tumor biology. Experientia 1992;48:1092-1102.

Van Wijk R. Biophoton and bio-communication. J Sci Explor 2001;15:183-197.

Voeikov VL, Novikov CN, Vilenskaya, ND. Low-level chemiluminescence of nondiluted human blood reveals its dynamic system properties. J Biomed Optics 1999;1:54-60.

Voeikov VL. Processes involving reactive oxygen species are the major source of structured energy for organizmal biophotonic field pumping. In: Beloussov L, Popp FA, Voeikov V, van Wijk $\mathrm{R}$, eds. Biophotons and Coherent Systems. Proceedings of the 2nd Alexander Gurwitsch Conference. Moscow: Moscow University Press, 2000.

Voeikov VL. Reactive oxygen species, water, photons and life. Rivista di Biologia/Biology Forum 2001a;94:237-258.

Voeikov VL. Mitogenetic radiation, biophotons and non-linear oxidative processes in aqueous media. In: Integrative Biophysics, Popp FA, Beloussov L, eds. Netherlands, Kluwer Academic Publishers, 2003a:331-359.

Voeikov VL. Biophoton research in blood reveals its holistic properties. Ind J Exp Biol 2003b:41:473-482.

Von Neumann J. Theory of Self-Reproducing Automata. (Ed. by A.W. Burks). Champaign, IL: University of Illinois Press, 1966.

Wackermann J, Seiter C, Keibel H, Walach H. Correlations between brain electrical activities of two spatially separated human subjects. Neurosci Lett 2003;336:60-64.

Walach H, Romer H. Complementarity is a useful concept for consciousness studies. A Reminder. Neuroendocrinol Lett 2000;21: 221-232.

Zhang BW. A hypothesis about the mechanism of acupuncture from the viewpoint of the connection between cosmos and human body. J Qingdao Coll Medicine 1961;1:70-75. 
Zhang CL, Popp FA. Log-normal distribution of physiological parameters and the coherence of biological systems. Med Hypotheses 1994;43:11-16.

Zhang CL. Electromagnetic standing waves as background of acupuncture system [in Chinese]. Acupunct Res 1996;4:52-62.

Zhang CL. Skin resistance vs. body conductivity. Frontier Perspect 2002;11:15-25.

Zhang CL. Electromagnetic body versus chemical body. Network 2003;81:7-10.
Zhu, ZX, et al.(1989). Biophysics for Acupuncture [in Chinese]. Beijing: Beijing Publishing House, 1989.

Address reprint requests to: Bruce D. Curtis, M.A., M.Div. 691 Fifth Avenue San Francisco, CA 94118 E-mail: brucedcurtis@earthlink.net 